\title{
Original
}

\section{La formación de los especialistas en Enfermería del Trabajo, una perspectiva internacional}

\section{The Training of Occupational Health Nursing, an International Perspective}

Javier González Caballero'

1. Instituto Nacional de la Seguridad Social. Bilbao. España.

Recibido: 04-05-2020

Aceptado: 23-05-2020

\section{Correspondencia}

Javier González Caballero

Instituto Nacional de la Seguridad Social.

Servicio de Prevención y Salud Laboral.

Gran Vía, 89-3․

48011 Bilbao.

944284500.

javier.gonzalez1@seg-social.es

\section{Resumen}

La Enfermería del Trabajo es una disciplina que aborda el estado de salud de los individuos en su relación con el medio laboral. Desde esta perspectiva, el artículo realiza una visión panorámica internacional de la formación de los futuros especialistas y analiza sus prioridades formativas posteriores. El análisis invita a una reflexión crítica y aboga por una armonización en los contenidos sin dejar de considerar las particularidades de las distintas realidades sociolaborales y profesionales.

Med Segur Trab (Internet). 2019;66(259):63-68

Palabras clave: Enfermería del Trabajo; Formación continua

\begin{abstract}
Occupational Health Nursing is a discipline that studies the health condition of individuals in relation to the workplace. From this perspective, the article provides an international overview of future specialists training and analyzes their subsequent training priorities. The analysis invites a critical reflection and advocates for a content harmonization of content while continuing to consider the particularities of different socio-occupational and professional realities.
\end{abstract}

Med Segur Trab (Internet). 2019;66(259):63-68

Keywords: Occupational Health Nursing; Professional training

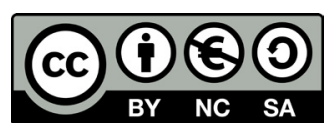

https://creativecommons.org/licenses/by-nc-sa/4.0/ 
En una visión panorámica internacional de esta disciplina enfermera, la formación de los especialistas en Enfermería del Trabajo muestra diferencias. En este contexto, diferentes asociaciones profesionales en el ámbito de la Unión Europea tienen definidas las competencias específicas de la Enfermería del Trabajo. De igual manera, países como Argentina $^{1,2}$, Australia ${ }^{3,4,5,6}$, Brasil ${ }^{7,8,9,10}$, Canadá ${ }^{11,12,13}$, Estados Unidos ${ }^{14,15,16,17,18,19,20,21,22}$, Japón ${ }^{23,24,25,26}$, Reino Unido ${ }^{27,28,29,30}$ y Nueva Zelanda ${ }^{31}$ entre otros, han realizado idéntico cometido. Con carácter diferenciado, las asociaciones norteamericanas poseen la facultad de acreditar como especialistas a aquellos profesionales que superen un examen establecido. En este supuesto, el cumplimiento de dos requisitos previos resulta indispensable: certificar tres mil horas de experiencia laboral desarrollando funciones como Enfermera del Trabajo y haber cursado una determinada formación en el ámbito de la especialidad, resultando preceptivo acreditar ambos requisitos en los cinco años previos a solicitar el reconocimiento como especialista.

Del análisis y desarrollo de las atribuciones profesionales de la Enfermería del Trabajo en los países citados se pueden extraer algunos aspectos relevantes. En unos casos es el periodo de formación de postgrado y la forma de acceso al mismo, en otros la definición, estructura y desarrollo de las propias competencias de la especialidad, y en los más, su acreditación y los órganos reguladores de la profesión son los que difieren con respeto a la Orden SAS/1348/2009 donde se definen las competencias transversales y específicas de Enfermería del Trabajo en España ${ }^{32}$. En este supuesto, el proceso de adquisición de competencias profesionales de los futuros especialistas denota algunas especificidades. Si bien en muchos países de nuestro entorno se ha optado por distintas modalidades, actualmente la capacitación se realiza como Enfermero Interno Residente (EIR) a través de un sistema de residencia en unidades docentes multidisciplinares acreditadas para la formación especializada. Un sistema al que se accede a través de una convocatoria anual de carácter estatal, siendo la duración establecida del periodo formativo de dos años en base a un programa consensuado por la Comisión Nacional de la especialidad de Enfermería del Trabajo ${ }^{(i)}$.

En las conclusiones del primer estudio realizado por la Federation of Occupational Health Nurses within the European Union (FOHNEU, 2004) donde se analizan los distintos programas de estudios de la especialidad impartidos y las competencias de mayor desarrollo en los quince estados participantes destacan las siguientes: la promoción de salud en los centros de trabajo, la prestación de primeros auxilios, la realización de exámenes de salud, la prevención de los accidentes laborales, la evaluación de los riesgos profesionales y el desarrollo de tareas administrativas ${ }^{33}$. La propia Federación publica un segundo informe (FOHNEU, 2012) donde participan seis países más que en la primera edición del trabajo, veintiuno en total. En su desarrollo, de nuevo describe las competencias de la especialidad en cada país y analiza los programas docentes que se imparten, observando una tendencia generalizada a que la formación especializada se realice a través de un máster universitario ${ }^{34}$.

En un mundo laboral cambiante, globalizado y en continua adaptación, FOHNEU evidencia que el envejecimiento de los especialistas, la aplicación de nuevas tecnologías y el estrés constituyen los tres riesgos más relevantes que destacan los profesionales ${ }^{34}$. El

(i) El EIR asiste a un programa docente durante veinticuatro meses constituido tanto por una formación teórica como práctica, incorporando la primera faceta la siguiente estructura: conceptos generales, marco conceptual y legislativo, técnicas de prevención de riesgos laborales y contenidos relacionados con las áreas de promoción de la salud, asistencia sanitaria, aspectos legales y periciales, gestión y docencia e investigación. En cuanto a la parte práctica se adquieren competencias en distintos contextos: atención especializada (rotación por los servicios de Urgencias hospitalarias, extrahospitalarias y traumatológicas; Alergología, Cardiología, Dermatología, Medicina preventiva, Neumología, Oftalmología, Otorrinolaringología, Radiodiagnóstico y Rehabilitación); atención primaria, atención continuada y una formación específica relacionada con los servicios de prevención, esta parcela constituye una prioridad fundamental del residente destinando doce meses del periodo de residencia. 
propio informe, en una descripción por países, señala que los estudios relacionados con la prevención y promoción de la salud son las principales áreas de investigación de la Enfermería del Trabajo en España; asimismo, alega como principal causa para desarrollar nuevos proyectos la falta de financiación.

Entre otras conclusiones de interés, el informe citado concluye que la presencia de servicios de prevención tanto en el ámbito público como privado está muy extendida dentro del área de estudio, la Unión Europea. Su existencia es obligatoria en países como Bélgica, Finlandia, Francia, Alemania, Grecia, Hungría, Holanda, Portugal, Eslovenia, España o Turquía, mientras que en Chipre y Rumanía solamente en el sector público. En otros países como Dinamarca, Irlanda, Malta, Suecia, Suiza y Reino Unido cuya constitución es voluntaria, disponen de una larga tradición en la presencia de este tipo de servicios. De forma concreta, Alemania, Bélgica, España, Finlandia, Francia, Grecia, Hungría, Portugal y Turquía tienen una disposición normativa específica que exige contratar especialistas en Enfermería del Trabajo ${ }^{34}$.

Por último, este relato de la presencia internacional de la especialidad se describe en el primer estudio mundial publicado en 2005 sobre las competencias de los profesionales vinculados a la salud laboral y del que no constan actualizaciones ${ }^{35}$. Definidas cuatro categorías de análisis en función del tipo de competencia y grado de desarrollo de los países participantes: enfermeras, médicos, ergonómos e higienistas industriales, el proyecto analizó 118 competencias en 89 estados. Entre sus conclusiones señala que el $66,70 \%$ de los países desarrollados disponen de titulación oficial y programa formativo específico en Enfermería del Trabajo. En sintonía, el propio estudio aporta un dato concluyente, en el $77,20 \%$ de los países desarrollados se han constituido sociedades de afines vs. el 36,70\% de aquellos en vías de desarrollo. Otro trabajo posterior donde participaron 450 especialistas en Enfermería del Trabajo procedentes de 17 países de los cinco continentes, eleva esta cifra hasta el $85,10 \%{ }^{36}$.

La especialidad demuestra un compromiso con la formación continuada ${ }^{37}$. En España prácticamente el $81 \%$ de las Enfermeras del Trabajo han cursado el nivel superior en prevención de riesgos laborales, destacando que casi el 50\% dispone de la titulación en todas las disciplinas preventivas contempladas en la Ley 31/1995 de Prevención de Riesgos Laborales ${ }^{38}$, siendo la Ergonomía y Psicosociología aplicada aquella que suscita más interés $(12,65 \%)$ frente al 35,30\% de un trabajo presentado por el Col-legi Oficial d'Infermeres $i$ Infermers de Barcelona ${ }^{39}$. En el referido estudio mundial realizado en colaboración con la Organización Mundial de la Salud, se cita un listado de las preferencias formativas en países desarrollados, entre ellas destacan los contenidos específicos relativos a la salud laboral y las normas que lo regulan. En el citado trabajo del Colegio profesional de Barcelona, la importancia se la conceden a los contenidos de patología laboral y gestión de la vigilancia de la salud; menor interés despiertan las áreas de informática, metodología de la investigación y estadística. Conclusiones que proporcionan una idea general de la motivación del especialista y que pudieran explicar el limitado desarrollo de la faceta investigadora en este campo por la escasa formación específica. No obstante, en un análisis pormenorizado de las competencias del colectivo enfermero muestra una circunstancia, los profesionales que han cursado formación superior en riesgos laborales, han contribuido en mayor medida a realizar comunicaciones científicas frente a quienes no disponen de esa acreditación formativa ${ }^{37}$. En esta misma línea, la Japan Society for Occupational Health apunta que casi el 19\% ha publicado un artículo en los últimos cinco años en una revista específica y cerca del $74 \%$ realiza entre una y seis actividades formativas al año, asumiendo en el $32 \%$ de los casos su coste ${ }^{25}$. Finalmente, aunque esta inquietud de formación es elevada, el 25\% de la profesión donde están representadas todas las especialidades, no considera prioritaria la formación específica para el desarrollo de sus competencias profesionales ${ }^{40}$. En este punto resulta significativa una de las conclusiones de un estudio donde establece que la mayor dificultad para asistir a este tipo de formación era de tipo de organizativo, relacionadas con la empresa contratante ${ }^{41}$, aspecto éste coincidente con otras especialidades enfermeras $^{42}$. 
El análisis panorámico de los distintos aspectos que determinan la formación del especialista permite una perspectiva global y puede resultar esclarecedor en un posible debate entre los agentes implicados, aspecto que quizá alumbre una armonización en los contenidos formativos generales y específicos considerando las particularidades de las distintas realidades sociolaborales y profesionales.

\section{AGRADECIMIENTOS}

A Jordi Delclós Clanchet, revisor de mirada pragmática. Gracias.

Bilbao, 22 mayo 2020.

\section{REFERENCIAS BIBLIOGRÁFICAS}

1. Tompkins O, Arce W. Occupational Health Nursing in Argentina. Workplace Health Saf 2012; 60 (10): 417-18. DOI: $10.1177 / 216507991206001002$.

2. Werner AF. Occupational health in Argentina. Int Arch Occup Environ Health 2000; 73 (5): 285-89. DOI: $10.1007 / \mathrm{s} 004200000154$.

3. Australian College of Occupational Health Nurses. Competency standards of Occupational Health Nurses. Rozelle, New South Wales, Australia: Australian College of Occupational Health Nurses, 1994.

4. Davey GD. Developing competency standards for occupational health nurses in Australia. AAOHN Journal 1995; 43 (3): 138-43. Erratum in: AAOHN Journal 1995; 43 (5): 275.

5. Davey GD, MacDonald-Taylor S, Clements S. Occupational health nurse practice in Australia. In: Education and training of Occupational Health Nurses. Scientific Committee on Occupational Health Nursing, 1996.

6. Mellor G, St John W, McVeigh C. Occupational health nursing practice in Australia: what occupational health nurses say they do and what they actually do. Collegian 2006; 13 (3): 18-24. DOI: 10.1016/s13227696(08)60528-4.

7. Cardoso MC, Douglas JL. Education of the nurses in workers health in Brazil. In: Education and training of Occupational Health Nurses. Scientific Committee on Occupational Health Nursing, 1996.

8. Marziale MHP, Hong OS, Morris JA, Rocha FLR. Los papeles y funciones del enfermero de salud laboral en Brasil y en los Estados Unidos de América. Rev. Latino-Am. Enfermagem [Internet] 2010 [acceso 26 Abr 2019]; 18 (2): [8 pantallas]. Disponible en: http://www.scielo.br/pdf/rlae/v18n2/es_07.pdf

9. Hong OS. Exploring Occupational Health Nursing in South America through brazilian experience. Workplace Health Saf 2012; 60 (3): 115-21. DOI: 10.1177/216507991206000306.

10. Roloff DIT, Cezar-Vaz MR, Bonow CA, Lautert L, Sant Anna CF, Couto AM. Occupational health nurses: interdisciplinary experience in occupational health. Rev Bras Enferm [Internet] 2016 [acceso 26 Abr 2019]; 69 (5): 842-855. Disponible en: http://www.scielo.br/scielo.php?pid=S0034-71672016000500897\&script= sci_arttext\&tlng=en

11. Verrall B. Occupational Health Nursing in Canada. Workplace Health Saf 2012; 60 (3): 111-13.

12. Canadian Nurses Association. Occupational Health Nurses Certification. Exam blueprint and specialty competencies. Canada: Canadian Nurses Association; 2013. [Acceso 27 Abr 2019]. Disponible en: https:// mycna.ca/-/media/nurseone/files/en/occupational_health_blueprint_and_competencies_e.pdf

13. Kilpatrick K, DiCenso A, Bryant-Lukosius D, Ritchie JA, Martin-Misener R, Carter N. Practice patterns and perceived impact of clinical nurse specialist roles in Canada: results of national survey. Int $\mathrm{J}$ Nurs Stud 2013; 50: 1524-36. DOI: 10.1016/j.ijnurstu.2013.03.005.

14. Burgel BJ, Wallace EM, Kemerer SD, Garbin M. Certified occupational health nursing. Job analysis in the United States. AAOHN Journal 1997; 45 (11): 581-91.

15. American Association of Occupational Health Nurses. Competencies in occupational health nursing AAOHN Journal 1999; 47 (12): 552-68.

16. Salazar MK, Kemerer S, Amann M, Fabrey LJ. Defining the roles and functions of occupational and enviromental health nurses: results of a national job analysis. AAOHN Journal 2002; 50 (1): 16-25.

17. American Association of Occupational Health Nurses. Competencies in occupational health nursing. AAOHN Journal 2003; 51 (7): 290-302

18. American Association of Occupational Health Nurses. Competencies in occupational and environmental health nursing. AAOHN Journal 2007; 55 (11): 442-47. 
19. Thompson MC, Wachs JE. Occupational health nursing in the United States. Workplace Health Saf 2012; 60 (3): 127-33. DOI: 10.1177/216507991206000308.

20. Thompson MC. Professional autonomy of occupational health nursing in the United States. Workplace Health Saf 2012; 60 (4): 159-65. DOI: 10.1177/216507991206000404.

21. American Association of Occupational Health Nurses. AAOHN Competencies. Workplace Health Saf 2015; 63 (11): 484-92. DOI: 10.1177/2165079915607876.

22. American Association of Occupational Health Nurses. Competencies in Occupational and Environmental Health Nursing. Workplace Health Saf 2015; 63 (11): 493-94. DOI: 10.1177/2165079915608192.

23. Kono K, Oku K. Education and training of occupational health nurses in Japan. In: Education and training of Occupational Health Nurses. Scientific Committee on Occupational Health Nursing, 1996.

24. Arino NS. Occupational health nursing in Japan: current practice and future perspectives. AAOHN Journal 2003; 51 (2): 58-64.

25. Mizuno-Lewis S, Kono K, Lewis DR, Gotoh Y, Hagi N, Sato M, Yoshikawa E, Higashikawa K, Yamazaki M, Naito M, Kondo N. Barriers to continuing education and continuing professional development among Occupational Health Nurses in Japan. Workplace Health Saf 2014; 62 (5): 198-205. DOI: $10.1177 / 216507991406200504$.

26. Kono K, Goto Y, Hatanaka J, Yoshikawa E. Competencies required for occupational health nurses. J Occup Health 2017; 59: 562-71. DOI: 10.1539/joh.16-0188-OA.

27. Raper JA. Occupational health nursing practice in the United Kingdom: the european influence. AAOHN Journal 1993; 41 (2): 84-9.

28. Royal College of Nursing. Education Occupational Health Nurses. An approach to align education with a service vision for occupational health nurses. London: Public Health England, 2016 [acceso 5 Dic 2019]. Disponible en: https://vivbennett.blog.gov.uk/wp-content/uploads/sites/90/2016/11/Educating-OHNsfinal-Oct-2016-FinalNB071116.pdf

29. Lalloo D et al. Core competencies for UK occupational health nurses: a Delphi study. Occup Med (Lond) 2016; 66 (8): 649-55. DOI: 10.1093/occmed/kqw089.

30. Lallo D, Demou E, Stevenson M, Gaffney M, Beaton E. Comparison of competency priorities between UK occupational physicians and occupational health nurses. Occup Environ Med 2017; 74: 384-86. DOI: 10.1136/oemed-2016-104049.

31. Lankhaar N. Development of a professional qualification and associated training program in New Zealand. AAOHN Journal 1991; 39 (7): 339-42.

32. Boletín Oficial del Estado. Orden SAS/1348/2009 de 6 de mayo por la que se aprueba y publica el programa formativo de la especialidad de Enfermería del Trabajo. BOE núm. 129 de 28/5/2009. Disponible en: http://www.boe.es/boe/dias/2009/05/28/pdfs/BOE-A-2009-8880.pdf

33. Federation of Occupational Health Nurses in the European Union. Occupational Health Nursing. Education and practice in the EU countries. Education Group, FOHNEU Final report 2004 [acceso 18 Oct 2019]. Disponible en: https://fohneu.org/images/pdf/OHNinEUfinalreport1.pdf

34. Federation of Occupational Health Nurses in the European Union. Occupational Health Nursing. Education, practice and profile in the EU countries. Education Group. Final report, 2012 [acceso 18 Oct 2019]. Disponible en: https://fohneu.org/images/pdf/final-report-on-OHN-education-practice-and-profile-2012.pdf

35. Delclós GL, Bright KA, Carson AI, Felknor SA, Mackey TA, Morandi MT, Schulze LJH, Whitehead LW. A global survey of occupational health competencies and curriculum. Int J Occup Environ Health $2005 ; 11$ (2): 185-98. DOI: 10.1179/oeh.2005.11.2.185.

36. Rogers B, Kono K, Palucci MH, Peurala M, Radford J, Staun J. International survey of Occupational Health Nurses roles in muldisciplinary teamwork in occupational health services. Workplace Health Saf 2014; 62 (7): 274-81. DOI: 10.1177/216507991406200702.

37. González J. Evolución y desarrollo de las competencias profesionales de Enfermería del Trabajo. Leioa (Bizkaia): Universidad del País Vasco/Euskal Herriko Universitatea; 2017.

38. Boletín Oficial del Estado. Ley 31/1995 de 8 de noviembre de Prevención de riesgos laborales. BOE núm. 269 de 10/11/1995. Disponible en: http://www.boe.es/boe/dias/1995/11/10/pdfs/A32590-32611.pdf

39. Col·legi Oficial d'Infermeria de Barcelona. Enquesta sobre la situació professional de les Infermeres/ers especialistes en Infermeria del Treball a la provincia de Barcelona a 2009. Barcelona (España), 2009.

40. Sindicato de Enfermería. Estudio SATSE. Percepción de estrés en los profesionales de enfermería en España. Madrid: Secretaría General Técnica SATSE, 2012.

41. Satomi ML, Keiko K, Desmond RL, Yuki G, Noriko H, Etsuko Y, Kaoru H, Masato Y, Masako N, Nobuko $\mathrm{K}$. Barriers to continuing education and continuing professional development among Occupational Health Nurses in Japan. Workplace Health Saf 2014; 62 (5): 198-205. DOI: 10.1177/216507991406200504. 
42. Llauradó M, Güell R, Castanera A, Sandalinas I, Argilaga E, Fortes del Valle ML, Jiménez MF, Bordonado L, Fuentes C. Barreras y motivaciones de los profesionales de enfermería para la utilización de la investigación en unidades de cuidados intensivos y en el servicio de emergencias médicas. Enferm Intensiva 2016; 27: 146-54. DOI: 10.1016/j.enfi.2016.05.001.

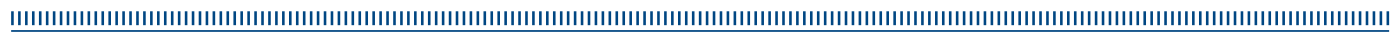

